\title{
Endoilluminator Light Source for Multiple Surgeries
}

\author{
Dr. K. S. Dhanalakshmi \\ Assistant Professor \\ Department of Electronics and \\ Communication Engineering \\ Kalasalingam Academy of Research and Education \\ Krishnan koil, Tamilnadu
}

\begin{abstract}
An endoilluminator is a surgical tool used to supply light for surgeons inside the body. Also known as a chandelier endo illuminator or chandelier probe, in vitreoretinal surgical treatment this kind of surgical lighting tool can assist surgeons better see their operating field and improve visualization of challenges that can also occur. The foremost objective of the challenge is the usage of endo illuminator mild for eye surgery to limit the complexity of dealing with eye surgery.
\end{abstract}

Keywords:- Arduino Uno, ATMEGA 328P IC, Luminous Light Source, SMPS, Power Supply.

\section{INTRODUCTION}

An endoilluminator is a surgical device used to supply mild for surgeon's inner the body. Also regarded as a chandelier endo illuminator or chandelier probe, in vitreoretinal surgical procedure this kind of surgical lighting fixtures tool can assist surgeons higher see their running subject and enhance visualization of challenges that may also occur. Some give up illuminators can be positioned into a cannula whilst others are inserted into the vitreous cavity. The predominant goal of the venture is the use of endo illuminator mild for eye surgery to reduce the complexity of dealing with eye surgery. Some of the endo illuminator tools has fixed brightness so that we need no longer be capable to change brightness. So that with the aid of adding brightness increment and decrement approach which assist the surgeons in the course of surgery. The wireless feature is additionally handy which can easily operate the gear thru cell or some sort of far off and additionally replacing buttons rather of touch display screen for incrementing and decrementing brightness. In this challenge the LED mild fiber plan $(0.5 \mathrm{~mm})$ for stabilizes the 23-25gauge tip in the eye and gives up to 20-25 lumen of measured illumination. Easy insertion and removal except suture placement make it convenient to exchange the function of the gadget during surgical operation and efficaciously shorten surgical time. This suture-free 2325 gauge endo illumination effectivity of vitreous surgery. For most surgical subspecialties, the key to illumination is brightness the brighter the better. The trouble for retinal surgeons are that, even though we would like our surgical surroundings to be brighter, we face the danger of retinal photo-toxicity, a threat unique to ophthalmologists and highest for retina surgeons. So we need to balance our desire for a brighter surgical discipline towards the hazard of developing retinal damage. One of the best benefits of

\author{
A Rohan, A Sreekanth, B Rakesh \\ Department of Electronics and \\ Communication Engineering \\ Kalasalingam Academy of Research and Education \\ Krishnan koil, Tamilnadu
}

the more modern USFDA approved led mild chips has been the introduction of clinically useful scientific lighted instruments.

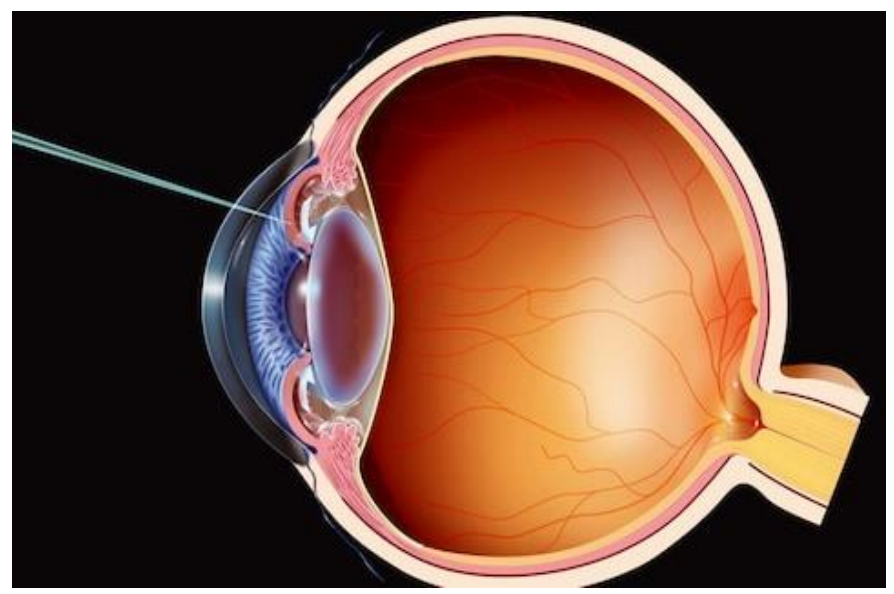

Fig 1:- Surgery image

\section{PROJECT AIM AND FOCUS}

The present endo illuminator equipment has some drawbacks which have an adapter only used for particular surgery, it may be ocular or optho surgeries. Due to this drawback we cannot use this equipment for multiple surgeries. Then the other drawback is based on LED which produces low lux (light intensity) due to this reason the surgeons cannot find the minute particles during eye surgeries. The older endoilluminator equipment's have a probe buttons mechanism which cannot give accurate light intensity. So the surgeons felt difficult to handle this type of mechanism during surgeries.

Which overcomes these existing drawbacks and provides new technology. We are using changeable adapters which can be used for multiple surgeries like ocular, optho surgeries etc. So, by changeable adapters surgeons can be benefited with using single equipment for multiple surgeries. It is connecting to the heat sink which produces some heat while the kit is working, so to reduce the heat we are placing cooling fans to exhaust the heat from the equipment. We are using LED which is nothing but luminous LED that produces high lux, due to that the surgeons can find minute particles during the surgeries. We are replacing the probe button mechanism with visual display (Touch Screen) that can help surgeons to increase or decrease the percentage of light intensity. We are using Arduino Uno with microcontroller of AT Mega 328p IC which is interfacing with 7 segment LCD screen or panel. 
Two SMPS are 5v 25Amp \& 5v 2Amp one is connecting to luminous LED and other is connect to Arduino respectively. The cooling fans are used to exhaust the heat.

\section{PROPOSED WORK}

The fundamental components are used in this project such as LED which is nothing but luminous LED that produces high lux, due to that the surgeons can discover minute particles during the surgeries and replacing the probe button mechanism with visual show (Touch Screen) that can help surgeons to increase or decrease the percentage of mild intensity and using wireless technology such as Bluetooth module. Arduino Uno with microcontroller of AT Mega 328p IC which is interfacing with 7 segment LCD screen or panel. Two SMPS are 5v 25Amp \&amp; 5v 2Amp one is connecting to luminous LED and other is connect to Arduino respectively. The cooling fans are used to exhaust the heat. The changeable adapters which can be used for more than one surgeries like ocular, optho surgeries etc. So, by changeable adapters surgeons can be benefited with using single tools for multiple surgeries.

\section{WORKING AND IMPLEMNTATION}

The working of the proposed project deals a lot of various topics. Let us put forth a bird eye view model of the project model and implementation. The constant power supply is used for equipment, Two SMPS (Switch Mode Power Supply) one is 5V 2AMP \& 5V 25AMP the first one is connect to the Arduino Uno which Arduino Uno need working voltage is $5 \mathrm{~V} 2 \mathrm{AMP}$ and second one is used for the Luminous LED actually Luminous LED required $4 \mathrm{~V}$ 2AMP by doing small changes in second SMPS that produce 4V 2AMP. By using touch screen display we can increase or decrease light intensity based on that Arduino Uno which have microcontroller ATMEGA 328P IC generate PWM (Pulse Width Modulation). If light is increase to 10 percent, then PWM ON 10 percent and OFF 90 percent similarly if it is increases to 20 percent the it ON 20 percent and OFF 80 percent in simple word 5Voltage is same in SMPS but current is increase from 0 to 25AMP, so that light intensity will increase. The changeable adapter is used for multiple surgeries and due to increasing intensity of LED the heat is generated in heat sink and also voltage fluctuation occurs so that we use RF filter is used to control and cooling fans are used to exhaust the heat from the equipment.

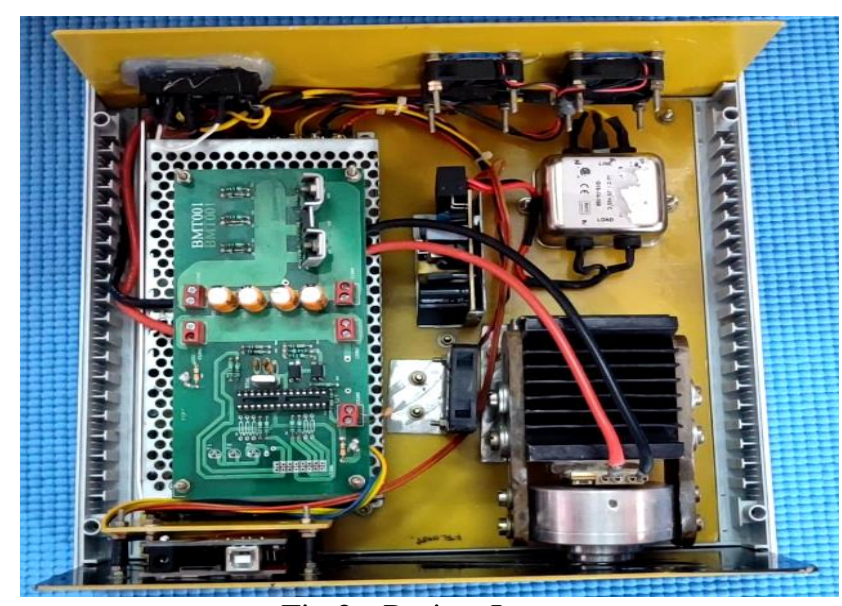

Fig 2:- Project Image

\section{FUNCTIONS}

$>$ Increment and decrement of light

$>$ Bluetooth for $\mathrm{ON}$ and $\mathrm{OFF}$

$>$ LCD for displaying percentage of light

$>$ Easy Implementation.

\section{BLOCK DIAGRAM}

There are nine leading aggregations in our project. The primary aggregation is the power supply block. This aggregation is the source of energy. Next aggregation is for Arduino Uno which is interface with 7 segment display and generate PWM. Next aggregation is Bluetooth module and Touch screen display. The final aggregation is the drive circuit, led module and current sensor which is used to control light intensity.

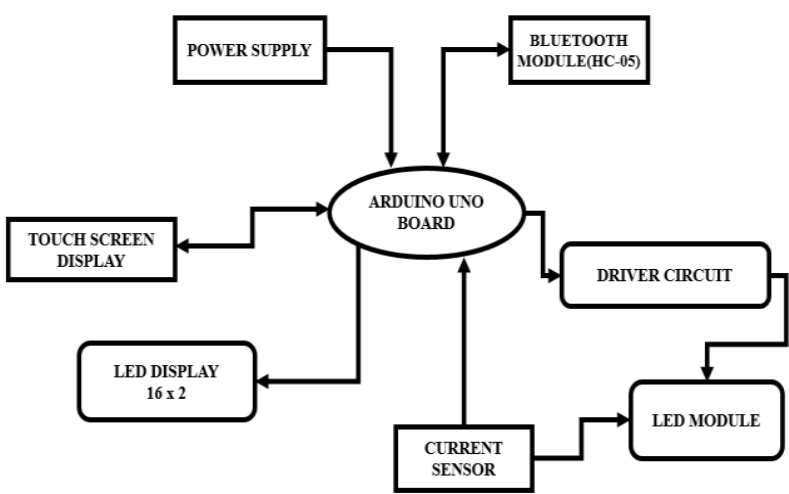

Fig 3:- Block diagram

VII. FUTURE SCOPE

In future we are going to propose our project by using IOT (Internet of Things) based, by using the IOT we can handle this equipment from any place and now we use Bluetooth module for only ON and OFF of the kit but we bring a wireless technology which can operate the equipment by using some sort of remote or mobile phone. In future we reduce the size of the kit by using SMT compatibility which can occupy less space and it can be easily handle by surgeons. 


\section{CONCLUSION}

The proposed system provides Output which the light we are using is luminous that can produce more lux which compare to normal lights used in present kit. Due to more lux the surgeons can see minute particles in eye during surgeries. The probe buttons are replaced with touch screen display by using that we can easily increase or decrease the light intensity and 7 segment display is used to show the percentage of light intensity. The SMPS which have $5 \mathrm{~V}$ 25AMP has been connected to LED light which is help to increase current due to that light brightness is increases. The changeable adapter is used for multiple surgeries it means the different surgeries we can use various adapters with same equipment it makes easy to the surgeons.

\section{REFERENCES}

[1]. Machemer R, Norton EW. A new concept for vitreous surgery. 3. Indications and results. Am J Ophthalmol 1972;74:1034-1056.

\section{http://informahealthcare.com/cot}

[2]. 1. Nishimura A, Kobayashi A, Segawa Y, Sugiyama K. Endoillumination-assisted cataract surgery in a patient with corneal opacity. J Cataract Refract Surg 2003;29:2277-80.

www.IJO.in

[3]. International Commission on Non-Ionizing Radiation Pro-tection. Guidelines on limits of exposure to broadband incoherent optical radiation (0.38 to $3 \mu \mathrm{m})$. Health Phys1997;73:539-54. https://bjo.bmj.com

[4]. Ahn SJ, Ahn J, Woo SJ, Park KH. Photoreceptor change and visual outcome after idiopathic epiretinal membrane removal with or without additional internal limiting membrane peeling. Retina. 2014;34(1):172181. https://www.dovepress.com

[5]. Erakgu ï T. Illumination systems. J Ret Vit 2007; 15:5-7.

[6]. Charles S. Illumination and phototoxicity issues in vitreoretinal surgery. Retina 2008;28:1-4.

[7]. Sakaguchi $\mathrm{H}$ and Oshima $\mathrm{Y}$. Considering the illuminationchoices in vitreoretinal surgery: continual improvementsallow for better, safer outcomes. Retinal Phys 2012; 3:26-31.

[8]. Koelbl PS, Lindner C, Lingenfelder C, et al. FaserloserMiniatur-Chandelier-LED-Endoilluminator fur die Pars-plana-Vitrektomie. Der Ophthalmologe 2016; 113: 47-51. 